\title{
Mutant BRAF feels the burn
}

Ketones are produced when the body runs low on glucose supplies and starts to burn fat, something that most of us aim for at the gym. This is not a metabolic pathway that one would associate with the proliferation of melanomas that express mutant BRAF; however, a paper published in Molecular Cell indicates that the production of ketones can promote the BRAF ${ }^{\mathrm{V} 600 \mathrm{E}}$-MEK-ERK oncogenic pathway and identifies a potential Achilles' heel.

Kang, Fan, Lin et al. used a short hairpin RNA (shRNA) library to target 1,361 of the 1,417 genes that encode proteins involved in metabolism and transduced human melanoma cell lines that expressed $\mathrm{BRAF}^{\mathrm{V} 600 \mathrm{E}}$ or NRAS ${ }^{\mathrm{Q} 61 \mathrm{~K} / \mathrm{R}}$ to identify metabolic factors for which knockdown limited the growth of $\mathrm{BRAF}^{\mathrm{V} 600 \mathrm{E}}$ cells only. The use of 3 different algorithms to identify the top hits produced 36 candidates, which

How is

HMGCL, an enzyme normally expressed by liver cells to induce ketogenesis, involved in BRAFV600 driven melanoma? ketone acetoacetate (AA) by HMGCL promotes BRAF ${ }^{\mathrm{V} 600 \mathrm{E}}$ signalling through the MEK1-ERK pathway but not the AKT-AMPK pathway. Moreover, they showed that AA directly promotes the interaction between $\mathrm{BRAF}^{\mathrm{V} 600 \mathrm{E}}$ and MEK1 and the subsequent phosphorylation of MEK1. They also showed that AA has no effect on the interaction of wild-type BRAF with MEK1.

How are $\mathrm{BRAF}^{\mathrm{V} 600 \mathrm{E}}$ and HMGCL linked? Knockdown of BRAF ${ }^{\mathrm{V} 600 \mathrm{E}}$, but not wild-type BRAF, resulted in reduced expression of HMGCL, reduced production of AA and compromised proliferation. This effect was not evident when MEK inhibitors were used, indicating that $\mathrm{BRAF}^{\mathrm{V} 600 \mathrm{E}}$, and not its downstream signalling pathways, increased the expression of HMGCL. In silico and luciferase analyses of the HMGCL promoter sequence indicated that there were binding sites for eight different transcription factors and chromatin immunoprecipitation indicated that OCT1-induced transcription of HMGCL was dependent on BRAF ${ }^{\mathrm{V} 600 \mathrm{E}}$ expression. Moreover, knockdown of OCT1 prevented the $\mathrm{BRAF}^{\mathrm{V} 600 \mathrm{E}}$-mediated upregulation of HMGCL.

The authors showed that the same pathway operates in hairy cell leukaemia, which express $\mathrm{BRAF}^{\mathrm{V} 600 \mathrm{E}}$, and that biopsy samples from patients with BRAF ${ }^{\mathrm{V} 600 \mathrm{E}}$

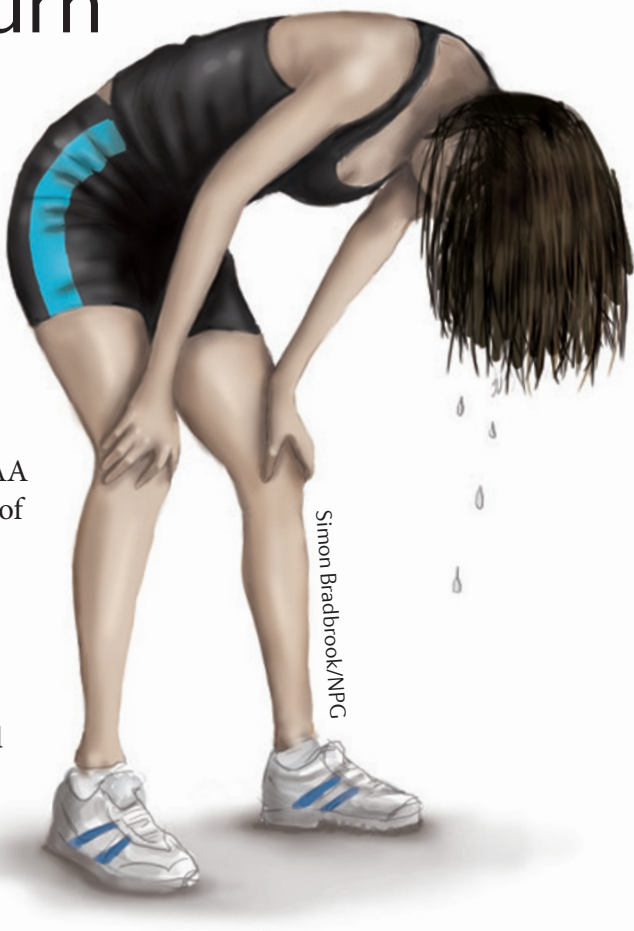

melanomas have increased levels of HMGCL expression. Moreover, knockdown of HMGCL compromises the growth of human $\mathrm{BRAF}^{\mathrm{V} 600 \mathrm{E}}$-mutant melanomas in immunocompromised mice. Additional work is required to determine how AA promotes the interaction between BRAF ${ }^{\mathrm{V} 600 \mathrm{E}}$ and MEK1 and whether this synthetic lethal interaction can be used to good effect in the clinic.

Nicola McCarthy Horizon Discovery Group, Cambridge, UK The author declares no competing interests.

ORIGINAL RESEARCH PAPER Kang, H.-B. Fan, J., Lin, R. et al. Metabolic rewiring by oncogenic BRAF V600E links ketogenesis pathway to BRAF-MEK1. Mol. Cell http://dx.doi. org/10.1016/j.molcel.2015.05.037 (2015) 